\title{
Tuberculous arthritis of the ankle joint masquerading as rheumatoid arthritis in a patient with lupus nephritis
}

\author{
Authors: Mohd N bin Ismail ${ }^{A}$ and Siti M Ab Rahim ${ }^{B}$
}

Tuberculosis is an airborne multisystemic disease which primarily infects the lungs. Isolated tuberculous arthritis is rare. We present a case of a 41-year-old woman with a known case of lupus nephritis class IV who complained of pain and swelling over her left ankle joint for several months. She was treated for rheumatoid arthritis and was started with immunosuppressive agents. However, she did not improve with treatment and was later diagnosed with tuberculous arthritis based on a molecular study from joint aspiration. She was started on antituberculosis medication and showed improvement. We highlight the challenges in reaching the diagnosis and the importance of including tuberculous arthritis in differential diagnoses in cases with persistent ankle swelling.

KEYWORDS: tuberculosis, tuberculous arthritis, ankle joint, extrapulmonary TB, rheumatoid arthritis

DOI: $10.7861 /$ clinmed.2020-0882

\section{Introduction}

Tuberculosis is a relatively common infectious disease and has remained a significant health problem throughout the world. Globally, it is estimated that 10 million people were infected with Mycobacterium tuberculosis in 2018 and there were more than 1.2 million deaths from tuberculosis worldwide. ${ }^{1}$

The symptoms of tuberculosis could be atypical and non-specific, especially in immunosuppressed patients. While patients with systemic lupus erythematosus (SLE) are at higher risk of developing opportunistic infection (including tuberculosis), isolated tuberculous arthritis of the ankle joint is rare. We present a case of a woman with underlying lupus nephritis class IV who complained of pain and swelling over the left ankle joint for several months. Otherwise, she didn't have any constitutional symptoms. Further workup confirmed that she had tuberculous arthritis. This case illustrates the difficulties to diagnose the disease in the presence of many overlapping features.

Authors: ${ }^{\text {A }}$ hysician, Hospital Sultanah Nur Zahirah, Terengganu, Malaysia; ${ }^{B}$ consultant rheumatologist, Hospital Sultanah Nur Zahirah, Terengganu, Malaysia

\section{Case presentation}

A 45-year-old woman presented with pain and swelling over her left ankle and right wrist joint for the previous 4 months. The swelling progressively increased in size and was associated with early morning stiffness. Otherwise, she denied any fever, cough, altered bowel habit or other systemic symptom and she denied any contact with a tuberculosis patient. She had a history of hypertension, uterine fibroid and class IV lupus nephritis. She was on regular diltiazem $60 \mathrm{mg}$ twice daily (bid), azathioprine $75 \mathrm{mg}$ once daily (od), hydroxychloroquine $100 \mathrm{mg}$ od, prednisolone $10 \mathrm{mg}$ od, alfacalcidol $0.25 \mu \mathrm{g}$ od and losartan $100 \mathrm{mg}$ od. In addition, she had taken mycophenolate mofetil tablets $1 \mathrm{~g}$ bid which she took for 1 year as the immunosuppressive agent for her lupus nephritis 2 years previously.

Clinical examination revealed swelling over the left ankle joint measuring $6 \times 8 \mathrm{~cm}$, as well as swelling over right wrist and second to fifth metacarpophalangeal joint over the right hand. Bedside ultrasound of the left foot revealed synovial hypertrophy with calcification within, as well as severe structural deformity and evidence of active synovitis. It also revealed joint erosion and effusion over right second to fifth metacarpophalangeal joint and bilateral wrist joints.

Total white cell count was $11.7 \times 10^{9} / \mathrm{L}$, C-reactive protein (CRP) was $31.4 \mathrm{mg} / \mathrm{L}$, erythrocyte sedimentation rate (ESR) was $48 \mathrm{~mm} /$ hour, and renal and liver function was normal. In view of her clinical presentation, she was treated for rheumatoid arthritis based on the 2010 American College of Rheumatology (ACR) criteria with a pending anti-citrullinated protein antibody (ACPA) test and was subsequently started with tablet methotrexate $15 \mathrm{mg}$ per week in addition to her previous medications. However, after 4 months on anti-inflammatory drugs, her left ankle didn't show any sign of improvement despite compliance to the treatment given. Magnetic resonance imaging (MRI) of her left ankle confirmed increased joint effusions with synovial proliferation especially in the intercarpal, carpal-metacarpal and subtalar joints with marked enhancement of the thickened synovium following intravenous contrast and extensive bone erosions (Fig 1). In view of her poor response to anti-inflammatory drugs and negative ACPA, the initial diagnosis of rheumatoid arthritis was revised.

Aspiration of the left ankle joint under ultrasound guidance was done and $10 \mathrm{cc}$ of greenish, cloudy fluid was aspirated. Synovial fluid cytology showed mainly neutrophils in the background of granular debris suggestive of acute suppurative inflammation. 


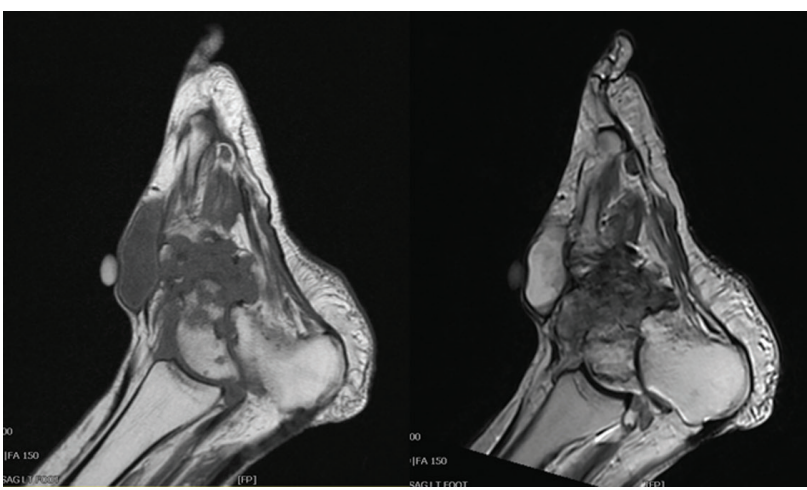

Fig 1. Magnetic resonance imaging of the left foot confirming increased joint effusions with synovial proliferation.

At that point of time, the patient was empirically treated for septic arthritis while waiting for other investigations and she was started on intravenous cefazolin. Tuberculin skin test showed a strong positive result with induration of $15 \mathrm{~mm}$ in diameter. After 5 days on antibiotics, the bacterial culture showed no growth, but molecular tuberculosis genome test turned out to be positive for Mycobacterium tuberculosis. She was subsequently started on a quadruple therapy of rifampicin, isoniazid, ethambutol and pyrazinamide for 2 months followed by maintenance phase comprising of isoniazid and rifampicin for 7 months.

The patient completed the 9 months of anti-tuberculosis drugs. At the same time, she was also referred to physiotherapy. Upon review, the symptoms improved greatly and the patient no longer complained of pain over the joint. The swelling markedly reduced in size; however, the left ankle deformity remains.

\section{Discussion}

Articular tuberculosis is a form of skeletal tuberculosis which accounts for approximately $1-3 \%$ of patients with tuberculosis, $50 \%$ of which were associated with pulmonary tuberculosis. ${ }^{2}$ It presents as a slowly progressive mono-arthritis involving weightbearing joints especially the spine, followed by the hip and knee joint. ${ }^{3}$ Ankle tuberculosis arthritis is rare, although the exact prevalence is unknown. The initial presentation is pain, followed by signs of inflammation for weeks. Fever and systemic symptoms are usually absent. ${ }^{4}$

It has been well-documented that SLE seems to be a risk factor for the development of tuberculosis and vice versa. Tuberculosis infection in SLE patients are more often extrapulmonary with more severe pulmonary involvement. ${ }^{5}$ In addition, the usage of immunosuppressive therapies in our patient augmented the risk of developing infection. ${ }^{6}$

This case reflects the diagnostic challenges faced in diagnosing tuberculous arthritis, especially in a patient with underlying connective tissue disease in view of many overlapping features. The diagnostic delay occurred because the diagnosis was thought to be rheumatoid arthritis or lupus-related inflammatory arthritis. Our patient was vaccinated with bacillus Calmette-Guérin (BCG) during childhood. She neither had any tuberculosis feature in any other organ nor had any past contact with tuberculosis patient. However, living in endemic region, the diagnosis of tuberculosis should always be taken into consideration.

The utilisation of MRI is very helpful in differentiating between tuberculous arthritis and rheumatoid arthritis, as uniform synovial thickening, large size of bone erosion, rim enhancement at site of bone erosion and extra-articular cystic masses were more frequent and more numerous in tuberculous arthritis. ${ }^{7}$ However, it is not diagnostic and warrants biopsy for confirmation.

\section{Conclusion}

We report a case of tuberculous arthritis involving the ankle joint that mimicked rheumatoid arthritis in a patient with lupus nephritis. The diagnosis of tuberculous arthritis is difficult in patients with SLE as there are many overlapping features in between the two. In addition, the typical presentation of tuberculosis might not be present, especially in an immunocompromised patient. Hence, the diagnosis of TB should always be considered in cases with chronic arthritis especially in endemic area.

\section{References}

1 World Health Organization. Global tuberculosis report: 2019. WHO, 2019. www.who.int/publications/i/item/global-tuberculosisreport-2019

2 Malaviya AN, Kotwal PP. Arthritis associated with tuberculosis. Best Pract Res Clin Rheumatolog 2003;17:319-43.

3 Golden MP, Vikram HR. Extrapulmonary tuberculosis: an overview. Am Fam Physician 2005;72:1761-8.

4 Pigrau-Serralach C, Rodriguez-Pardo D. Bone and joint tuberculosis. Eur Spine J 2013;22(Suppl 4):556-66.

5 Balbi GGM, Machado-Ribeiro F, Marques CDL et al. The interplay between tuberculosis and systemic lupus erythematosus. Curr Opin Rheumatol 2018;30:395-402

6 Insoo Kang, Sung Hwan Park. Infectious complications in SLE after immunosuppressive therapies. Curr Opin Rheumatol 2003;15: 528-34.

7 Choi JA, Koh SH, Hong SH et al. Rheumatoid arthritis and tuberculous arthritis: differentiating MRI features. Am J Roentgenol 2009;193: 1347-53.

Address for correspondence: Dr Mohd Noor bin Ismail, Department of General Medicine, Hospital Sultanah Nur Zahirah, Jalan Sultan Mahmud, 20400 Kuala Terengganu, Malaysia.

Email: muhammadnoor.ismail@gmail.com

Twitter: @muhammad_noor1 